\title{
UNIFORM ANALYTIC-GEVREY REGULARITY OF SOLUTIONS TO A SEMILINEAR HEAT EQUATION
}

\author{
TODOR GRAMCHEV \\ Dipartimento di Matematica e Informatica, Università di Cagliari \\ via Ospedale 72, 09124 Cagliari, Italy \\ E-mail:todor@unica.it \\ GRZEGORZ ŁYSIK \\ Institute of Mathematics, Polish Academy of Sciences \\ Sniadeckich 8, 00-956 Warszawa 10, Poland \\ \& Świętokrzyska Academy, Kielce, Poland \\ E-mail: lysik@impan.gov.pl
}

\begin{abstract}
We study the Gevrey regularity down to $t=0$ of solutions to the initial value problem for a semilinear heat equation $\partial_{t} u-\Delta u=u^{M}$. The approach is based on suitable iterative fixed point methods in $L^{p}$ based Banach spaces with anisotropic Gevrey norms with respect to the time and the space variables. We also construct explicit solutions uniformly analytic in $t \geq 0$ and $x \in \mathbb{R}^{n}$ for some conservative nonlinear terms with symmetries.
\end{abstract}

1. Introduction. We consider the initial value problem for a semilinear heat equation

$$
\partial_{t} u-\Delta u=u^{M},\left.\quad u\right|_{t=0}=u_{0},
$$

where $\Delta$ is the Laplace operator on $\mathbb{R}^{n}$ and $M$ is a positive integer. The initial data will be supposed analytic or more generally, belonging to some Gevrey space $G^{\sigma}$. Broadly speaking, the main aim of the present paper is to study in detail simultaneously the analytic-Gevrey regularity with respect to $t \geq 0$ and $x \in \mathbb{R}^{n}$.

We construct a new, as far as we know, functional framework consisting of scales of Banach spaces of functions, having anisotropic Gevrey regularity $G^{\tau, \sigma}$ with respect to $t \geq 0, x \in \mathbb{R}^{n}$ and derive precise estimates in such scales for the solutions of the IVP

2000 Mathematics Subject Classification: Primary 35B65, 35E15, 35K05, 35K15.

Key words and phrases: semilinear heat equation, initial value problem, Gevrey spaces.

Research of the first author supported by NATO grant PST.CLG.979347, and GNAMPAINDAM, Italy. Research of the second author supported by NATO grant PST.CLG.979347, and EU FP6 MCA-ToK SPADE2, MTKD-CT-2004-014508 and MNiSW SPB-M, Poland.

The paper is in final form and no version of it will be published elsewhere. 
down to $t=0$. It is well known that for the heat equation one has in general $\tau \geq 2 \sigma$ (in fact, if $\sigma=1$ it was M. Gevrey who derived in 1918 what we will call nowadays $G^{1,2}\left(\left[0,+\infty\left[\times \mathbb{R}_{x}^{n}\right)\right.\right.$ estimates for the heat kernel, cf. [9]).

In the linear case we derive sharp estimates on the uniform anisotropic regularity in $t \geq 0, x \in \mathbb{R}^{n}$ down to $t=0$ and we allow initial data in subspaces of entire functions of exponential type $1 /(1-\sigma), \sigma \in] 0,1[$. Next, we dwell upon the Banach algebra properties of such scales when the Gevrey index $\sigma \geq 1$. The Banach algebra properties allow us to show local existence and uniqueness by means of standard Picard iteration scheme combined with the contraction principle. Finally, inspired by the celebrated Oseen solution for the 2-D Navier-Stokes equation, we exhibit classes of semilinear equations with conservative nonlinear terms with symmetries admitting uniformly analytic solutions with respect to $t \geq 0$ and $x \in \mathbb{R}^{n}$, provided the initial conditions admit suitable symmetries.

Our motivation is based on functional-analytic methods and it deals primarily with the analytic regularization of the heat operator with respect to the space variables for positive times for various classes of semilinear parabolic equations, the Burgers' equation, the Navier-Stokes equation etc. We refer to the pioneering paper of Foiaş and Temam [7] for the Navier-Stokes equation with periodic data, while concerning semilinear parabolic equations, H. Aikawa and N. Hayashi proved in [1] that if $u_{0} \in L^{p}\left(\mathbb{R}^{n}\right)$ and $f$ is a polynomial of degree $\leq 1+2 p / n$ then for $t>0$ the solution $u(t, \cdot)$ of $\partial_{t} u=\Delta u+$ $f(u), u(0, \cdot)=u_{0}$ extends analytically to a strip in $\mathbb{C}^{n}$ with width proportional to $\sqrt{t}$. Later on, results for more general semilinear parabolic equations have been proved (cf. [24], [6], [3], [4], [13] and the references therein). We stress that the aforementioned papers do not deal with the Gevrey type regularity in $t$ down to $t=0$. One of the main technical difficulties is more pertinent to the nonlinear analysis, namely suitable nonlinear superposition estimates for Gevrey anisotropic spaces.

There are also results for analyticity with respect to the time variable, but only in a conic neighborhood of $t=0$. The first result in this direction was obtained by $\mathrm{S}$. Öuchi [21] who proved analyticity in time in a sector $\left\{t=r e^{i \theta}, 0<r<\infty,|\theta|<\alpha\right\}$ under the assumption that $f(u)$ is a monotone non-increasing polynomial and the initial function is bounded and continuous. We mention also the paper of Z. Grujić and I. Kukavica [14] for similar results for the Kuramoto-Sivashinsky equation. It appears however that the solution to (1) need not be analytic in time at zero even if the initial data is globally analytic (see [18]).

We can summarize our approach as follows: in order to address the issues of the uniform regularity in $t \geq 0$ and $x \in \mathbb{R}^{n}$ we develop a new functional analytic framework which enables us to deal simultaneously with the regularity with respect to $t \geq 0$ and $x \in \mathbb{R}^{n}$.

2. Banach spaces of uniformly Gevrey functions. Let $\Omega \subset \mathbb{R}^{n}$ be an open domain and let $\sigma>0$. We define $G_{u n}^{\sigma}(\Omega)$, the space of uniformly Gevrey functions of index $\sigma$, as the set of all $f \in C^{\infty}(\Omega)$ such that there exists $C<\infty$ satisfying

$$
\sup _{x \in \Omega}\left|\partial_{x}^{\alpha} f(x)\right| \leq C^{|\alpha|+1} \alpha !^{\sigma}, \quad \alpha \in \mathbb{N}_{0}^{n} .
$$


where $\alpha !=\alpha_{1} ! \ldots \alpha_{n} !, \alpha=\left(\alpha_{1}, \ldots, \alpha_{n}\right) \in \mathbb{N}_{0}^{n}$. Given $f \in G_{u n}^{\sigma}\left(\mathbb{R}^{n}\right)$, in view of (2), we can define

$$
\rho_{\sigma}[f]=\sup \left\{C^{-1}>0:(2) \text { holds }\right\} .
$$

Clearly, if $\sigma=1$ then every $f \in G_{u n}^{1}\left(\mathbb{R}^{n}\right)$ extends to a holomorphic function in $\{z=$ $\left.\left(z_{1}, \ldots, z_{n}\right) \in \mathbb{C}^{n} ; \max _{j=1, \ldots, n}\left|\operatorname{Im} z_{j}\right|<\rho_{1}[f]\right\}$.

Local Gevrey spaces $G^{\sigma}(\Omega)$ are defined in a natural way as the projective limit of $G_{u n}^{\sigma}(\widetilde{\Omega})$ over an exhausting sequence of open sets $\widetilde{\Omega}$ relatively compact in $\Omega$. Thus $f \in$ $G^{\sigma}(\Omega)$ iff for every $\widetilde{\Omega} \Subset \Omega$ one can find $C>0$ such that $(2)$ holds with $\Omega$ replaced by $\widetilde{\Omega}$. In particular, if $\sigma=1$ we recover the well known set $G^{1}(\Omega)=A(\Omega)$ of real analytic functions on $\Omega$ while for $\sigma>1$ the $G^{\sigma}(\Omega)$ admits nonzero compactly supported functions. We refer to cf. [23], [19] for more details on Gevrey spaces with index $\sigma \geq 1$. We point out that the Gevrey spaces $G^{\sigma}, \sigma \geq 1$, are a natural framework for the study of PDEs with multiple characteristics and questions of regularity of solutions to evolution PDEs of Mathematical Physics, see [19] and the references therein.

The case of $0<\sigma<1$ has not been dealt with in the literature on PDEs. In that case the space $G_{u n}^{\sigma}(\Omega)$ consists of restrictions to $\Omega$ of functions from the space $\mathcal{O}^{\exp }\left(\mathbb{C}^{n} ; \rho\right)$ of entire functions of exponential order $\rho=1 /(1-\sigma)$, i.e. satisfying the estimate

$$
|F(z)| \leq C \exp \left\{L|z|^{\rho}\right\} \text { for } z \in \mathbb{C}^{n}
$$

with some $C<\infty$ and $L<\infty$ (cf. [17] for the one dimensional case). Typically, in applications, one introduces scales of Banach spaces depending on a parameter $\rho>0$,

$$
G^{\sigma}(\rho, X)=\left\{u \in X: \partial^{\alpha} u \in X \text { for any } \alpha \in \mathbb{N}_{0}^{n} \text { and }\|u\|_{\sigma, \rho ; X}<\infty\right\},
$$

where

$$
\|u\|_{\sigma, \rho ; X}:=\sum_{\alpha \in \mathbb{N}_{0}^{n}} \frac{\rho^{|\alpha|}}{(\alpha !)^{\sigma}}\left\|\partial^{\alpha} u\right\|_{X},
$$

and $X$ is some Banach space of function on $\Omega$ (e.g., $X$ might be $C^{k}(\bar{\Omega})$ or a Sobolev space $\left.H_{p}^{k}(\Omega), 1 \leq p \leq \infty, k \geq 0\right)$. We set $\rho_{\sigma}(u)=\sup \left\{\rho>0: u \in G^{\sigma}(\rho, X)\right\}$, cf. [2] and the references therein.

In order to deal with functions which have different behavior in time and space directions we introduce scales of anisotropic Gevrey spaces.

Let $T, \sigma, \tau, \rho, \theta$ be positive numbers and let $X$ be a Banach space of functions on $\Omega$ and $Y=L^{\infty}([0, T] ; X)$ equipped with the norm $\|u\|_{Y}=\sup _{t \in] 0, T[}\|u(t, \cdot)\|_{X}$. Set

$$
G^{\tau, \sigma}(\theta, \rho, Y)=\left\{u \in Y: \partial_{t}^{l} \partial_{x}^{\alpha} u \in Y \text { for any } l \in \mathbb{N}_{0}, \alpha \in \mathbb{N}_{0}^{n} \text { and }|u|_{\tau, \sigma, \theta \rho ; Y}<\infty\right\},
$$

where

$$
|u|_{\tau, \sigma, \theta, \rho ; Y}:=\sum_{l=0}^{\infty} \sum_{\alpha \in \mathbb{N}_{0}^{n}} \frac{\theta^{l} \rho^{|\alpha|}}{(l !)^{\tau}(\alpha !)^{\sigma}} \sup _{t \in] 0, T[}\left\|\partial_{t}^{l} \partial_{x}^{\alpha} u(t, \cdot)\right\|_{X} .
$$

Usually in order to prove regularity results for nonlinear equations one first proves good estimates for solutions to non-homogeneous linear equations. In the case of the spaces $G^{\tau}$ and $G^{\tau, \sigma}$ we could only prove the estimates with a loss of regularity. Namely we have 
Proposition 2.1 (cf. [10], Prop. 3.1 and 3.2). Let $X=H_{p}^{k}\left(\mathbb{R}^{n}\right), 1 \leq p \leq \infty, k \in \mathbb{N}_{0}$, $Y=L^{\infty}([0, T] ; X), 0<T \leq \infty$. Assume that $u_{0} \in G^{\sigma}\left(\rho_{0}, X\right), g \in G^{\tau, \sigma}\left(\theta, \rho_{0} ; Y\right)$. Then the solution $u$ of

$$
\partial_{t} u=\Delta u+g,\left.\quad u\right|_{t=0}=0, \quad t>0, x \in \mathbb{R}^{n},
$$

belongs to $G^{\tau, \sigma}(\theta, \rho ; Y)$ for any $\rho<\rho_{0}, \theta>0$ if $\tau>2 \sigma$ and for $\rho<\rho_{0}, 0<\theta<\theta_{\max }:=$ $\left(4^{\sigma} n C^{2}\right)^{-1}$ if $\tau=2 \sigma$, where $C=(2 / \epsilon)^{\sigma} \cdot 1 / \rho_{0}$ if $\rho=(1-\epsilon)^{\sigma} \rho_{0}$. Moreover

$$
\|u\|_{\tau, \sigma, \theta, \rho ; Y} \leq K\left\|u_{0}\right\|_{\sigma, \rho_{0} ; X}+K(T+\theta)\|g\|_{\tau, \sigma, \theta_{0}, \rho_{0} ; Y}
$$

where with $\tau=2 \sigma+\delta$,

$$
\begin{aligned}
& K=2^{n} \exp \left\{n \delta\left(2 \cdot 4^{\sigma} n \theta C^{2}\right)^{1 / \delta}\right\} \quad \text { if } \quad \delta>0, \\
& K=\left(1-4^{\sigma} n \theta C^{2}\right)^{-n} \quad \text { if } \quad \delta=0,0<\theta<\theta_{\text {max }} .
\end{aligned}
$$

Note that the above Proposition does not hold for $\rho_{0}=\rho$. It resembles the so-called tamed estimates in Nash-Moser or KAM type methods for spaces of analytic functions. For that reason we cannot apply it in the method of successive approximations in solving a semilinear heat equation.

3. Global uniform Gevrey spaces $\widetilde{G}$. The purpose of this section is to introduce a new type of norms suitable for the simultaneous study of the uniform anisotropic critical $G^{2 \sigma, \sigma}$ Gevrey regularity in $x \in \mathbb{R}^{n}$ and near $t=0$. The main advantage of the use of the new norms is that, in contrast to Proposition 2.1, no loss "à la Nash-Moser" type tamed estimates occurs.

Let $X$ be a Banach space of functions on $\mathbb{R}^{n}$. We define

$$
\widetilde{G}^{\sigma}(\rho ; X)=\left\{u \in X: \partial_{x}^{\alpha} u \in X \text { for any } \alpha \in \mathbb{N}_{0}^{n} \text { and } E^{\sigma, \rho ; X}[u]<\infty\right\},
$$

where

$$
E^{\sigma, \rho ; X}[u]:=\sum_{\alpha \in \mathbb{N}_{0}^{n}} \frac{\rho^{|\alpha|}}{\Gamma(\sigma|\alpha|+1)}\left\|\partial_{x}^{\alpha} u\right\|_{X} .
$$

Analogously for $0<T<\infty$ we define $\widetilde{G}^{\tau, \sigma}(\theta, \rho ; Y)=\left\{u \in Y=L^{\infty}([0, T] ; X): \partial_{t}^{l} \partial_{x}^{\alpha} u \in\right.$ $Y$ for any $l \in \mathbb{N}_{0}, \alpha \in \mathbb{N}_{0}^{n}$ and $\left.E^{\tau, \sigma, \theta \rho ; Y}[u]<\infty\right\}$, where

$$
E^{\tau, \sigma, \theta, \rho ; Y}[u]:=\sum_{l=0}^{\infty} \sum_{\alpha \in \mathbb{N}_{0}^{n}} \frac{\theta^{l} \rho^{|\alpha|}}{\Gamma(\tau l+\sigma|\alpha|+1)}\left\|\partial_{t}^{l} \partial_{x}^{\alpha} u\right\|_{Y} .
$$

The relation between the spaces $G$ and $\widetilde{G}$ is given by the following Proposition 3.1 (cf. [10], Prop. 4.1). Let $\sigma, \rho>0$ and $u \in G^{\sigma}(\rho ; X)$. Then $u \in \widetilde{G}^{\sigma}(\widetilde{\rho} ; X)$ for any $\widetilde{\rho}<\sigma^{\sigma} \rho$ and

$$
E^{\sigma, \widetilde{\rho} ; X}[u] \leq C_{\sigma, \widetilde{\rho} / \rho}\|u\|_{\sigma, \rho ; X}
$$

with some $C_{\sigma, \tilde{\rho} / \rho}<\infty$.

Proposition 3.2 (cf. [10], Prop. 4.4). Let $\sigma \geq 1, \rho>0$ and $\beta \in \mathbb{N}_{0}^{n}$. If $u \in \widetilde{G}^{\sigma}(\rho, X)$ then $\partial^{\beta} u \in \widetilde{G}^{\sigma}(\widetilde{\rho}, X)$ for any $\widetilde{\rho}<\rho$ and

$$
E^{\sigma, \tilde{\rho} ; X}\left[\partial^{\beta} u\right] \leq C^{|\beta|} \Gamma(\sigma|\beta|+1) \cdot E^{\sigma, \rho ; X}[u]
$$

where $C=C_{1}^{\sigma} / \rho$ with $C_{1}=1+\left((\rho / \tilde{\rho})^{1 / \sigma}-1\right)^{-1}$. 
4. Gevrey estimates for a linear heat equation in $\widetilde{G}$ spaces. Now we shall prove two propositions about the solutions of a linear heat equation in the spaces $\widetilde{G}$. Although the IVP for the heat equation is well known it seems that the estimates proposed in Propositions 4.1 and 4.2 below are a novelty.

Proposition 4.1. Let $X=H_{p}^{k}\left(\mathbb{R}^{n}\right), 1 \leq p \leq \infty, k \in \mathbb{N}_{0}, Y=L^{\infty}([0, T] ; X), 0<T \leq \infty$ and let $u_{0} \in \widetilde{G}^{\sigma}(\rho ; X), \sigma, \rho>0$. Then the solution $u=e^{t \Delta} u_{0}$ of

$$
\partial_{t} u=\Delta u,\left.\quad u\right|_{t=0}=u^{0}, \quad t>0, x \in \mathbb{R}^{n},
$$

belongs to $\widetilde{G}^{\tau, \sigma}(\theta, \rho ; Y)$ for any $\theta>0$ if $\tau>2 \sigma$ and for $0<\theta<\theta_{\max }=\rho^{2} / n$ if $\tau=2 \sigma$. Moreover

$$
E^{\tau, \sigma, \theta, \rho ; Y}[u] \leq K E^{\sigma, \rho ; X}\left[u_{0}\right],
$$

if $\tau>2 \sigma$, with some $K=K\left(\tau, \sigma, \theta / \rho^{2}, n\right)$ and for the critical case $\tau=2 \sigma$

$$
E^{2 \sigma, \sigma, \theta, \rho ; Y}[u] \leq K E^{\sigma, \rho ; X}\left[u_{0}\right] \quad \text { if } \theta<\rho^{2} / n
$$

with $K=\left(1-n \theta / \rho^{2}\right)^{-n}$.

Proof. Let $E_{n}(t, x)$ be the heat kernel

$$
E_{n}(t, x)=(4 \pi t)^{-n / 2} \exp \left\{-x^{2} / 4 t\right\} .
$$

Then $u(t, x)=E_{n}(t, \cdot) * u_{0}(x)$ and $\left\|E_{n}(t, \cdot)\right\|_{L^{1}}=1$. Hence the Young inequality gives $\left\|\partial^{\alpha} u(t, \cdot)\right\|_{L^{p}} \leq\left\|\partial^{\alpha} u_{0}\right\|_{L^{p}}$. So we can reduce the proof to the case $X=L^{p}\left(\mathbb{R}^{n}\right)$. Then clearly $u \in Y=L^{\infty}([0, T] ; X)$ with $\|u\|_{Y} \leq\left\|u_{0}\right\|_{X}$. Next for any $l \in \mathbb{N}_{0}$ and $\alpha \in \mathbb{N}_{0}^{n}$

$$
\partial_{t}^{l} \partial_{x}^{\alpha} u(t, x)=\Delta_{x}^{l} \partial_{x}^{\alpha} u(t, x)=\sum_{\ell \in \mathbb{N}_{0}^{n},|\ell|=l}\left(\begin{array}{l}
l \\
\ell
\end{array}\right) \partial_{x}^{\alpha+2 \ell} u(t, x) .
$$

Hence

$$
\left\|\partial_{t}^{l} \partial_{x}^{\alpha} u(t, \cdot)\right\|_{X} \leq \sum_{\ell \in \mathbb{N}_{0}^{n},|\ell|=l}\left(\begin{array}{l}
l \\
\ell
\end{array}\right)\left\|\partial_{x}^{\alpha+2 \ell} u_{0}\right\|_{X}
$$

and

$$
\left\|\partial_{t}^{l} \partial_{x}^{\alpha} u\right\|_{Y} \leq \sum_{\ell \in \mathbb{N}_{0}^{n},|\ell|=l}\left(\begin{array}{l}
l \\
\ell
\end{array}\right)\left\|\partial_{x}^{\alpha+2 \ell} u_{0}\right\|_{X}
$$

Therefore, after the change of the summation index $\beta=\alpha+2 \ell$ we get

$$
\begin{aligned}
E^{\tau, \sigma, \theta, \rho ; Y}[u] & \leq \sum_{l=0}^{\infty} \sum_{\alpha \in \mathbb{N}_{0}^{n}} \sum_{\ell \in \mathbb{N}_{0}^{n},|\ell|=l} \frac{\theta^{l} \rho^{|\alpha|}}{\Gamma(\tau l+\sigma|\alpha|+1)}\left(\begin{array}{c}
l \\
\ell
\end{array}\right)\left\|\partial_{x}^{\alpha+2 \ell} u_{0}\right\|_{X} \\
& \leq \sum_{\beta \in \mathbb{N}_{0}^{n}}\left(\sum_{\ell \leq \beta / 2} \frac{1}{\Gamma(\sigma|\beta|+(\tau-2 \sigma) l+1)}\left(\begin{array}{l}
l \\
\ell
\end{array}\right) \frac{\theta^{l}}{\rho^{2 l}}\right) \rho^{|\beta|}\left\|\partial_{x}^{\beta} u_{0}\right\|_{X} \\
& \leq \sum_{\beta \in \mathbb{N}_{0}^{n}} Q_{\tau, \sigma}(\beta) \frac{\rho^{|\beta|}}{\Gamma(\sigma|\beta|+1)}\left\|\partial^{\beta} u_{0}\right\|_{X},
\end{aligned}
$$

where

$$
Q_{\tau, \sigma}(\beta)=\sum_{2 \ell \leq \beta} \frac{\Gamma(\sigma|\beta|+1)}{\Gamma(\sigma|\beta|+(\tau-2 \sigma) l+1)}\left(\begin{array}{l}
l \\
\ell
\end{array}\right) \frac{\theta^{l}}{\rho^{2 l}}, \quad \beta \in \mathbb{N}_{0}^{n}
$$


We shall prove that one can find a constant $K=K\left(\tau, \sigma, \theta / \rho^{2}, n\right)<\infty$ such that for any $\beta \in \mathbb{N}_{0}^{n}$

$$
Q_{\tau, \sigma}(\beta) \leq K
$$

In order to prove (20) assume first that $\tau=2 \sigma$. Then

$$
Q_{2 \sigma, \sigma}(\beta)=\sum_{2 \ell \leq \beta}\left(\begin{array}{l}
l \\
\ell
\end{array}\right)\left(\frac{\theta}{\rho^{2}}\right)^{l} \leq \sum_{\ell \in \mathbb{N}_{0}^{n}}\left(\frac{n \theta}{\rho^{2}}\right)^{l}=\left(\frac{1}{1-n \theta / \rho^{2}}\right)^{n} .
$$

So in that case

$$
K\left(2 \sigma, \sigma, \theta / \rho^{2}, n\right)=\left(\frac{1}{1-n \theta / \rho^{2}}\right)^{n}<\infty \quad \text { for } \quad 0<\theta<\rho^{2} / n .
$$

Now let $\sigma>2 \tau$. By the Stirling formula there exists a constant $C(\sigma)<\infty$ such that

$$
\frac{\Gamma(z+1)}{\Gamma(z+\zeta+1)} \leq C(\sigma) \frac{e^{\zeta}}{(z+\zeta)^{\zeta}} \quad \text { for } \quad z \geq \sigma, \quad \zeta \geq 0
$$

Hence

$$
\frac{\Gamma(\sigma|\beta|+1)}{\Gamma(\sigma|\beta|+(\tau-2 \sigma) l+1)} \leq C(\sigma) \frac{e^{(\tau-2 \sigma) l}}{(\sigma|\beta|+(\tau-2 \sigma) l)^{(\tau-2 \sigma) l}}, \quad|\beta| \geq 1 .
$$

Therefore we get

$$
\begin{aligned}
Q_{\tau, \sigma}(\beta) & \leq C(\sigma) \sum_{2 \ell \leq \beta} \frac{e^{(\tau-2 \sigma) l}}{(\sigma|\beta|+(\tau-2 \sigma) l)^{(\tau-2 \sigma) l}}\left(\begin{array}{c}
l \\
\ell
\end{array}\right)\left(\frac{\theta}{\rho^{2}}\right)^{l} \\
& \leq C(\sigma) \sum_{2 \ell \leq \beta}\left(\frac{n \theta e^{(\tau-2 \sigma)}}{\rho^{2}(\sigma|\beta|+(\tau-2 \sigma) l)^{(\tau-2 \sigma)}}\right)^{l} \\
& \leq C(\sigma)\left\{\sum_{l=0}^{|\beta| / 2}\left(\frac{n \theta e^{(\tau-2 \sigma)}}{\rho^{2}(\sigma|\beta|+(\tau-2 \sigma) l)^{(\tau-2 \sigma)}}\right)^{l}\right\}^{n} .
\end{aligned}
$$

For $\delta=\tau-2 \sigma>0, A=n e^{\delta} \theta / \rho^{2}$, put

$$
R(s)=\sum_{l=0}^{s / 2}\left(\frac{A}{(\sigma s+\delta l)^{\delta}}\right)^{l}, \quad s \in \mathbb{N}_{0} .
$$

Then $R(0)=R(1)=1$. Next if $2 \leq s<\frac{1}{\sigma}(2 A)^{1 / \delta}$ then

$$
\begin{aligned}
R(s) & \leq 1+\frac{A}{(s \sigma)^{\delta}}+\ldots+\left(\frac{A}{(s \sigma)^{\delta}}\right)^{\lfloor s / 2\rfloor} \\
& \leq\left(\frac{s}{2}+1\right) \cdot \max \left\{1,\left(\frac{A}{(2 \sigma)^{\delta}}\right)^{\lfloor s / 2\rfloor}\right\} .
\end{aligned}
$$

Finally if $s \geq \frac{1}{\sigma}(2 A)^{1 / \delta}$ then $A /(s \sigma)^{\delta} \leq 1 / 2$ and $R(s) \leq 2$. Hence one can find a constant $C(A, \sigma, \delta)$ such that $R(s) \leq C(A, \sigma, \delta)$ for any $s \in \mathbb{N}_{0}$. Combining this with (19) and (22) we get $(20)$ with $K\left(\tau, \sigma, \theta / \rho^{2}, n\right)=C(\sigma) \cdot C^{n}(A, \sigma, \delta)$ where $\delta=\tau-2 \sigma, A=n e^{\delta} \theta / \rho^{2}$. Finally (18) and (20) gives (15) and (16) which completes the proof. 
Proposition 4.2. Let $X=H_{p}^{k}\left(\mathbb{R}^{n}\right), 1 \leq p \leq \infty, k \in \mathbb{N}_{0}, Y=L^{\infty}([0, T] ; X), 0<T<\infty$. Let $g \in \widetilde{G}^{\tau, \sigma}(\theta, \rho ; Y)$ and let $u$ satisfy

$$
\partial_{t} u=\Delta u+g,\left.\quad u\right|_{t=0}=0, \quad t>0, x \in \mathbb{R}^{n},
$$

Then $u \in \widetilde{G}^{\tau, \sigma}(\theta, \rho ; Y)$ for any $\theta>0$ if $\tau>2 \sigma$ and for $0<\theta<\theta_{\max }=\rho^{2} / n$ if $\tau=2 \sigma$. Moreover

$$
E^{\tau, \sigma, \theta, \rho ; Y}[u] \leq K(T+\theta) E^{\tau, \sigma, \theta, \rho ; Y}[g]
$$

where $K=K\left(\tau, \sigma, \theta / \rho^{2}, n\right)<\infty$ for any $\theta>0$ if $\tau>2 \sigma$ and $K\left(2 \sigma, \sigma, \theta / \rho^{2}, n\right)=$ $\left(1-n \theta / \rho^{2}\right)^{-n}<\infty$ for $0<\theta<\theta_{\max }$ if $\tau=2 \sigma$.

Proof. As in the proof of Proposition 4.1 we can assume that $X=L^{p}\left(\mathbb{R}^{n}\right)$. We have

$$
u(t, x)=\int_{0}^{t} E_{n}(t-s, \cdot) * g(s, \cdot)(x) d s .
$$

Since $\left\|E_{n}(t-s, \cdot)\right\|_{L^{1}}=1$ the Young inequality gives $\|u(t, \cdot)\|_{X} \leq \int_{0}^{t}\|g(s, \cdot)\|_{X} d s$. Hence

$$
\|u\|_{Y} \leq\|g\|_{L^{1}([0, T] ; X)} \leq T\|g\|_{Y} .
$$

Next note that for any $l \in \mathbb{N}_{0}$

$$
\partial_{t}^{l} u=\Delta_{x}^{l} u+\sum_{j=0}^{l-1} \Delta_{x}^{j} \partial_{t}^{l-1-j} g \quad \text { and } \quad \Delta_{x}^{l} u=\sum_{\ell \in \mathbb{N}_{0}^{n},|\ell|=l}\left(\begin{array}{l}
l \\
\ell
\end{array}\right) \partial_{x}^{2 \ell} u .
$$

So by the Young inequality for any $l \in \mathbb{N}_{0}, \alpha \in \mathbb{N}_{0}^{n}$

$$
\begin{aligned}
\left\|\partial_{t}^{l} \partial_{x}^{\alpha} u(t, \cdot)\right\|_{X} \leq & \sum_{\ell \in \mathbb{N}_{0}^{n},|\ell|=l}\left(\begin{array}{l}
l \\
\ell
\end{array}\right) \int_{0}^{t}\left\|\partial_{x}^{\alpha+2 \ell} g(s, \cdot)\right\|_{X} d s \\
& +\sum_{j=0}^{l-1}\left\|\Delta_{x}^{j} \partial_{x}^{\alpha} \partial_{t}^{l-1-j} g(t, \cdot)\right\|_{X} .
\end{aligned}
$$

Hence for any $l \in \mathbb{N}_{0}, \alpha \in \mathbb{N}_{0}^{n}, \partial_{t}^{l} \partial_{x}^{\alpha} u \in Y$ and

$$
\left\|\partial_{t}^{l} \partial_{x}^{\alpha} u\right\|_{Y} \leq T \sum_{\ell \in \mathbb{N}_{0}^{n},|\ell|=l}\left(\begin{array}{l}
l \\
\ell
\end{array}\right)\left\|\partial_{x}^{\alpha+2 \ell} g\right\|_{Y}+\sum_{j=0}^{l-1}\left\|\Delta_{x}^{j} \partial_{x}^{\alpha} \partial_{t}^{l-1-j} g\right\|_{Y} .
$$

Now we decompose $E^{\tau, \sigma, \theta, \rho ; Y}[u]$ according to the above inequality,

$$
\begin{aligned}
E^{\tau, \sigma, \theta, \rho ; Y}[u] \leq & T \sum_{l=0}^{\infty} \sum_{\alpha \in \mathbb{N}_{0}^{n}} \frac{\theta^{l} \rho^{|\alpha|}}{\Gamma(\tau l+\sigma|\alpha|+1)} \sum_{\ell \in \mathbb{N}_{0}^{n},|\ell|=l}\left(\begin{array}{l}
l \\
\ell
\end{array}\right)\left\|\partial_{x}^{\alpha+2 \ell} g\right\|_{Y} \\
& +\sum_{l=0}^{\infty} \sum_{\alpha \in \mathbb{N}_{0}^{n}} \frac{\theta^{l} \rho^{|\alpha|}}{\Gamma(\tau l+\sigma|\alpha|+1)} \sum_{j=0}^{l-1}\left\|\Delta_{x}^{j} \partial_{x}^{\alpha} \partial_{t}^{l-1-j} g\right\|_{Y} \\
=: & T I_{1}+I_{2} .
\end{aligned}
$$

Following the proof of Proposition 4.1 we get

$$
I_{1} \leq K E^{\tau, \sigma, \theta, \rho ; Y}[g] .
$$


Next we estimate $I_{2}$ :

$$
\begin{aligned}
I_{2} & =\sum_{j=0}^{\infty} \sum_{\alpha \in \mathbb{N}_{0}^{n}} \sum_{l=j+1}^{\infty} \frac{\theta^{l} \rho^{|\alpha|}}{\Gamma(\tau l+\sigma|\alpha|+1)}\left\|\partial_{t}^{l-1-j} \Delta_{x}^{j} \partial_{x}^{\alpha} g\right\|_{Y} \\
& =\sum_{l=0}^{\infty} \sum_{\alpha \in \mathbb{N}_{0}^{n}} \sum_{m=0}^{\infty} \frac{\theta^{l+1+m} \rho^{|\alpha|}}{\Gamma(\tau(l+1+m)+\sigma|\alpha|+1)}\left\|\partial_{t}^{m} \Delta_{x}^{l} \partial_{x}^{\alpha} g\right\|_{Y} \\
\leq & \sum_{\ell \in \mathbb{N}_{0}^{n}} \sum_{\alpha \in \mathbb{N}_{0}^{n}} \sum_{m=0}^{\infty} \frac{\theta^{l+1+m} \rho^{|\alpha|}}{\Gamma(\tau(l+1+m)+\sigma|\alpha|+1)}\left(\begin{array}{l}
l \\
\ell
\end{array}\right)\left\|\partial_{t}^{m} \partial_{x}^{\alpha+2 \ell} g\right\|_{Y} \\
\leq & \sum_{m=0}^{\infty} \sum_{\beta \in \mathbb{N}_{0}^{n}}\left\{\sum_{\ell \leq \beta / 2} \frac{\theta^{l+1} / \rho^{2 l} \cdot \Gamma(\tau m+\sigma|\beta|+1)}{\Gamma(\tau(l+1+m)+\sigma(|\beta|-2 l)+1)}\left(\begin{array}{l}
l \\
\ell
\end{array}\right)\right\} \\
& \times \frac{\theta^{m} \rho^{|\beta|}}{\Gamma(\tau m+\sigma|\beta|+1)}\left\|\partial_{t}^{m} \partial_{x}^{\beta} g\right\|_{Y} \\
\leq & \theta \cdot \sum_{m=0}^{\infty} \sum_{\beta \in \mathbb{N}_{0}^{n}} Q(m, \beta) \frac{\theta^{m} \rho^{|\beta|}}{\Gamma(\tau m+\sigma|\beta|+1)}\left\|\partial_{t}^{m} \partial_{x}^{\beta} g\right\|_{Y} \\
\leq & K \theta E^{\tau, \sigma, \theta, \rho ; Y}[g]
\end{aligned}
$$

since $\Gamma(x+a) \Gamma(b+a) \leq \Gamma(x+b+a) \Gamma(a)$ for $a>0, b>0, x \geq 0$ and so

$$
Q(m, \beta):=\sum_{\ell \leq \beta / 2} \frac{\Gamma(\tau m+\sigma|\beta|+1)}{\Gamma(\tau m+\sigma|\beta|+(\tau-2 \sigma) l+1)}\left(\begin{array}{l}
l \\
\ell
\end{array}\right) \frac{\theta^{l}}{\rho^{2 l}} \leq Q(\beta) \leq K .
$$

Finally $E^{\tau, \sigma, \theta, \rho ; Y}[u] \leq T I_{1}+I_{2} \leq K(T+\theta) E^{\tau, \sigma, \theta, \rho ; Y}[g]$.

5. Nonlinear estimates in Gevrey spaces. In order to deal with semilinear equations in $\widetilde{G}$ spaces we need precise estimations of nonlinear superpositions.

Proposition 5.1. Let $\sigma, \rho$ be positive numbers. If $X$ is $C^{k}(\bar{\Omega}), k \in \mathbb{N}_{0}$ or the Sobolev space $H_{p}^{s}(\Omega)$ for $1 \leq p \leq \infty, s>n / p$ then $\widetilde{G}^{\sigma}(\rho, X)$ is a Banach algebra for $\sigma \geq 1$. Moreover,

$$
E^{\sigma, \rho ; X}[u v] \leq \omega E^{\sigma, \rho ; X}[u] E^{\sigma, \rho ; X}[v]
$$

where $\omega=\omega(X)$ is the Schauder constant for $X$.

Proof. By the Schauder lemma $\|u v\|_{X} \leq \omega\|u\|_{X}\|v\|_{X}$ for $u, v \in X$. Next for $u, v \in$ $\widetilde{G}^{\sigma}(\rho, X)$ we estimate

$$
\begin{aligned}
E^{\sigma, \rho ; X}[u v] & =\sum_{\alpha \in \mathbb{N}_{0}^{n}} \frac{\rho^{|\alpha|}}{\Gamma(\sigma|\alpha|+1)}\left\|\sum_{\beta \leq \alpha}\left(\begin{array}{c}
\alpha \\
\beta
\end{array}\right) \partial^{\beta} u \cdot \partial^{\alpha-\beta} v\right\|_{X} \\
& \leq \omega \sum_{\alpha \in \mathbb{N}_{0}^{n}} \frac{\rho^{|\alpha|}}{\Gamma(\sigma|\alpha|+1)} \cdot \sum_{\beta \leq \alpha}\left(\begin{array}{c}
\alpha \\
\beta
\end{array}\right)\left\|\partial^{\beta} u\right\|_{X} \cdot\left\|\partial^{\alpha-\beta} v\right\|_{X} \\
& =\omega \sum_{\beta \in \mathbb{N}_{0}^{n}} \frac{\rho^{|\beta|}\left\|\partial^{\beta} u\right\|_{X}}{\Gamma(\sigma|\beta|+1)} \cdot \sum_{\alpha \geq \beta} \rho^{|\alpha-\beta|}\left(\begin{array}{c}
\alpha \\
\beta
\end{array}\right) \frac{\Gamma(\sigma|\beta|+1)}{\Gamma(\sigma|\alpha|+1)}\left\|\partial^{\alpha-\beta} v\right\|_{X}
\end{aligned}
$$




$$
=\omega \sum_{\beta \in \mathbb{N}_{0}^{n}} \frac{\rho^{|\beta|}\left\|\partial^{\beta} u\right\|_{X}}{\Gamma(\sigma|\beta|+1)} \cdot \sum_{\gamma \in \mathbb{N}_{0}^{n}} \frac{\rho^{|\gamma|}\left\|\partial^{\gamma} v\right\|_{X}}{\Gamma(\sigma|\gamma|+1)} \cdot C_{\sigma}(\beta, \gamma)
$$

where

$$
C_{\sigma}(\beta, \gamma)=\frac{(\beta+\gamma) !}{\beta ! \gamma !} \cdot \frac{\Gamma(\sigma|\beta|+1) \Gamma(\sigma|\gamma|+1)}{\Gamma(\sigma|\beta+\gamma|+1)}, \quad \beta, \gamma \in \mathbb{N}_{0}^{n}
$$

We shall prove that $C_{\sigma}(\beta, \gamma) \leq 1$ for any $\beta, \gamma \in \mathbb{N}_{0}^{n}$ if $\sigma \geq 1$ which implies (27). Clearly for $\sigma=1$ we have

$$
C_{1}(\beta, \gamma)=\left(\begin{array}{c}
\beta+\gamma \\
\beta
\end{array}\right) /\left(\begin{array}{c}
|\beta+\gamma| \\
|\beta|
\end{array}\right) \leq 1
$$

So it is sufficient to show that for any $k, l \in \mathbb{N}_{0}$ the function

$$
F_{k, l}(\sigma)=\frac{\Gamma(\sigma k+1) \Gamma(\sigma l+1)}{\Gamma(\sigma(k+l)+1)}
$$

is non-increasing for $\sigma \geq 1$. Since $F_{k, l}(\sigma)=1$ if $k=0$ or $l=0$ we can assume that $k \geq 1$ and $l \geq 1$. By the properties of the Euler $\Gamma$ and $B$ functions we have

$$
F_{k, l}(\sigma)=(\sigma(k+l)+1) \cdot B(\sigma k+1, \sigma l+1)=(\sigma(k+l)+1) \cdot \int_{0}^{1} t^{\sigma k}(1-t)^{\sigma l} d t .
$$

Hence

$$
\frac{d F_{k, l}(\sigma)}{d \sigma}=\int_{0}^{1}\{k+l+(\sigma(k+l)+1)(k \ln t+l \ln (1-t))\} t^{\sigma k}(1-t)^{\sigma l} d t .
$$

We shall show that the expression in parentheses is non-positive for any $0<t<1$ which implies $\frac{d F_{k, l}(\sigma)}{d \sigma} \leq 0$ proving our claim. To this end note that since $k \ln t+l \ln (1-t)<0$ we can assume $\sigma=1$ and so we need to show that

$$
\frac{k+l}{k+l+1} \leq-k \ln t+l \ln (1-t) \quad \text { for any } 0<t<1 .
$$

Next note that the function $f(t)=-k \ln t-l \ln (1-t), 0<t<1$, assumes a minimum at the point $t=k /(k+l)$ equal to $(k+l) \ln (k+l)-k \ln k-l \ln l$. So (28) reduces to

$$
\frac{k+l}{k+l+1} \leq(k+l) \ln (k+l)-k \ln k-l \ln l \quad \text { for } \quad k, l \in \mathbb{N} .
$$

Finally the left hand side of $(29)$ is $<1$ while the right one is $>1$.

REMARK 5.2. If $0<\sigma<1$ the $E$ norm of $u v$ cannot be estimated by that of $u$ and $v$ without the loss of $\rho$.

The next proposition generalizes Proposition 5.1 to the case of the power function.

Proposition 5.3. Let $X$ be $C^{k}(\bar{\Omega}), k \in \mathbb{N}_{0}$ or the Sobolev space $H_{p}^{s}(\Omega)$ for $1 \leq p \leq \infty$, $s>n / p$ with $\omega \geq 1$ being its Schauder constant. Let $M \in \mathbb{N}, M \geq 2$. Then we have

$$
\begin{aligned}
E^{\sigma, \rho ; X}\left[u^{M}\right] & \leq \omega^{M-1}\left(E^{\sigma, \rho ; X}[u]\right)^{M}, \\
E^{\sigma, \rho ; X}\left[u^{M}-v^{M}\right] & \leq \omega^{M-1}\left(\max \left\{E^{\sigma, \rho ; X}[u], E^{\sigma, \rho ; X}[v]\right\}\right)^{M-1} \cdot E^{\sigma, \rho ; X}[u-v]
\end{aligned}
$$

for all $u, v \in \widetilde{G}^{\sigma}(\rho ; X)$ with $\sigma \geq 1, \rho>0$. Analogously

$$
E^{\tau, \sigma, \theta, \rho ; Y}[f(u)] \leq \omega^{M-1}\left(E^{\tau, \sigma, \theta, \rho ; Y}[u]\right)^{M},
$$




$$
\begin{aligned}
E^{\tau, \sigma, \theta, \rho ; Y}\left[u^{M}-v^{M}\right] \leq & \omega^{M-1}\left(\max \left\{E^{\tau, \sigma, \theta, \rho ; Y}[u], E^{\tau, \sigma, \theta, \rho ; Y}[v]\right\}\right)^{M-1} \\
& \times E^{\tau, \sigma, \theta, \rho ; Y}[u-v]
\end{aligned}
$$

for all $u, v \in \widetilde{G}^{\tau, \sigma}(\theta, \rho ; Y)$ with $\tau, \sigma \geq 1$ and $\theta, \rho>0$.

Proof. Clearly (30) follows immediately from Proposition 5.1, while (31) is a consequence of that proposition and the equality

$$
u^{M}-v^{M}=(u-v) \sum_{i+j=M-1} u^{i} v^{j} .
$$

Next (32) and (33) hold by an analogue of Proposition 5.1 for the spaces $\widetilde{G}^{\tau, \sigma}(\theta, \rho ; Y)$.

6. The main result. We have

Theorem 6.1. Let $M \in \mathbb{N}, M \geq 2$ and $\sigma \geq 1, \tau \geq 2 \sigma, \rho, \theta>0$. Let $X$ be the Sobolev space $H_{p}^{k}\left(\mathbb{R}^{n}\right)$ for $1 \leq p \leq \infty, k \in \mathbb{N}, k>n / p$. Assume that $u_{0} \in \widetilde{G}^{\sigma}(\rho ; X)$. Then the solution to the initial value problem

$$
\partial_{t} u=\Delta u+u^{M},\left.\quad u\right|_{t=0}=u_{0},
$$

belongs to $\widetilde{G}^{\tau, \sigma}(\theta, \rho ; Y)$ provided that $(T+\theta)\left(E^{\sigma, \rho ; X}\left[u_{0}\right]\right)^{M-1}$ is small enough and $0<$ $\theta<\rho^{2} / n$ if $\tau=2 \sigma$.

Proof. Define the approximation scheme by

$$
\begin{aligned}
\partial_{t} U_{N+1} & =\Delta U_{N+1}+U_{N}^{M}, \\
U_{N+1}(0, x) & =u_{0}(x), \quad N \in \mathbb{N}_{0},
\end{aligned}
$$

with $U_{0}=e^{t \Delta} u_{0}$. Then by Propositions 4.1 and 4.2 we get

$$
\begin{aligned}
E^{\tau, \sigma, \theta, \rho ; Y}\left[U_{0}\right] & \leq K E^{\sigma, \rho ; X}\left[u_{0}\right], \\
E^{\tau, \sigma, \theta, \rho ; Y}\left[U_{N+1}\right] & \leq K E^{\sigma, \rho ; X}\left[u_{0}\right]+K(T+\theta) E^{\tau, \sigma, \theta, \rho ; Y}\left[U_{N}^{M}\right], \quad N \in \mathbb{N}_{0},
\end{aligned}
$$

for any $\theta>0$ if $\tau>2 \sigma$ and for $0<\theta<\rho^{2} / n$ if $\tau=2 \sigma$. Next if $\tau, \sigma \geq 1$ by Proposition 5.3 we can find a constant $C<\infty$ depending on $\omega$ and $M$ such that

$$
E^{\tau, \sigma, \theta, \rho ; Y}\left[U_{N}^{M}\right] \leq C\left(E^{\tau, \sigma, \theta, \rho ; Y}\left[U_{N}\right]\right)^{M} .
$$

Hence combining (35) with (36) we get

$$
E^{\tau, \sigma, \theta, \rho ; Y}\left[U_{N+1}\right] \leq K E^{\sigma, \rho ; X}\left[u_{0}\right]+C K(T+\theta)\left(E^{\tau, \sigma, \theta, \rho ; Y}\left[U_{N}\right]\right)^{M} .
$$

Now we apply the following

Claim. If

$$
2 K L\left(2 K C_{0}\right)^{M-1} \leq 1
$$

and

$$
C_{N+1} \leq K C_{0}+K L C_{N}^{M} \quad \text { for } \quad N \in \mathbb{N}_{0}
$$

then

$$
C_{N} \leq\left(2-2^{-N}\right) K C_{0}<2 K C_{0} \quad \text { for } \quad N \in \mathbb{N}_{0} .
$$


Proof. Clearly (40) holds for $N=0$. Next assuming (40) we get by (39) and (38)

$$
\begin{aligned}
C_{N+1} & \leq K C_{0}+C_{N} \cdot K L C_{N}^{M-1} \\
& \leq K C_{0}+C_{N} / 2 \leq\left(2-2^{-N-1}\right) K C_{0}<2 K C_{0} .
\end{aligned}
$$

Applying the Claim with $L=C(T+\theta), C_{0}=E^{\sigma, \rho ; X}\left[u_{0}\right]$ and $C_{N}=E^{\tau, \sigma, \theta, \rho ; Y}\left[U_{N}\right]$ we conclude that $U_{N}$ belongs to a ball in $\widetilde{G}^{\tau, \sigma}(\theta, \rho ; Y)$ of radius $2 K C_{0}$ provided that $(T+\theta)\left(2 K C_{0}\right)^{M-1} \leq 1 /(2 C K)$. Now for $N \in \mathbb{N}$ put $V_{N}=U_{N}-U_{N-1}$. Then $V_{N}$ satisfies (with $U_{-1} \equiv 0$ )

$$
\partial_{t} V_{N}=\Delta V_{N}+U_{N-1}^{M}-U_{N-2}^{M}, \quad V_{N}(0, x)=0, \quad N \in \mathbb{N} .
$$

By Propositions 4.2 and 5.3 we get with $D=\omega^{M-1}<\infty$

$$
\begin{aligned}
E^{\tau, \sigma, \theta, \rho ; Y}\left[V_{N}\right] \leq & K(T+\theta) E^{\tau, \sigma, \theta, \rho ; Y}\left[U_{N-1}^{M}-U_{N-2}^{M}\right] \\
\leq & K(T+\theta) E^{\tau, \sigma, \theta, \rho ; Y}\left[V_{N-1}\right] \\
& \times \omega^{M-1}\left(\max \left\{E^{\tau, \sigma, \theta, \rho ; Y}\left[U_{N-1}\right], E^{\tau, \sigma, \theta, \rho ; Y}\left[U_{N-2}\right]\right\}\right)^{M-1} \\
\leq & K(T+\theta) D\left(2 K C_{0}\right)^{M-1} \cdot E^{\tau, \sigma, \theta, \rho ; Y}\left[V_{N-1}\right] .
\end{aligned}
$$

Hence if $K(T+\theta) D\left(2 K C_{0}\right)^{M-1}<1$ the sequence $U_{N}=U_{0}+\sum_{j=1}^{N} V_{j}$ converges to a function $u \in \widetilde{G}^{\tau, \sigma}(\theta, \rho ; Y)$. Clearly $u$ satisfies (34).

7. Examples of analyticity down to $t=0$ and stability properties. The aim of this section is to exhibit solutions for semilinear parabolic equations with conservative terms defined by rotation type vector fields which is an analogue to the celebrated Oseen solution to the 2-D Navier-Stokes equation (see [20]) in 1911.

We consider semilinear parabolic equations of the following type

$$
\partial_{t} u-\Delta u=X\left(u^{M}\right), \quad t>0, x \in \mathbb{R}^{n},
$$

where

(43) $X$ is a polynomial vector field tangential to $S_{r}=\left\{x \in \mathbb{R}^{n}:|x|=r\right\}, r>0$.

EXAMPLE 7.1. Vector fields of the type $Q(x)\left(x_{j} \partial_{k}-x_{k} \partial_{j}\right), 1 \leq j<k \leq n, Q(x)$ being a polynomial, are tangent to $S_{r}, r>0$.

Before the formulation of the next theorem recall that a function $\varphi$ belongs to the Gelfand-Shilov space $S_{\nu}^{\mu}\left(\mathbb{R}^{n}\right)$ iff one can find $C<\infty, D<\infty$ and $a>0$ such that for any $\alpha \in \mathbb{N}_{0}^{n}$

$$
\left|\partial^{\alpha} \varphi(x)\right| \leq C D^{|\alpha|} \alpha !^{\mu} \exp \left\{-a|x|^{1 / \nu}\right\} \text { for } x \in \mathbb{R}^{n}
$$

(cf. Gelfand-Shilov [8], Ch. IV). We consider the initial data

$$
u(0, x)=u_{0}(x)=\theta\left(|x|^{2}\right),
$$

where $\theta$ is a real valued continuous function on $[0, \infty)$. Then we have

Theorem 7.2. Let $\theta$ be a real valued continuous function on $[0, \infty)$. Suppose that $\theta(y)$ grows infraexponentially for $y \rightarrow+\infty$, i.e., for every $\varepsilon>0$ one can find $C_{\varepsilon}>0$ such that

$$
|\theta(y)| \leq C_{\varepsilon} e^{\varepsilon y}, \quad y \geq 0 .
$$


Then the IVP (42), (45) has a unique solution $u(t, x)$

$$
u(t, x)=e^{t \Delta} u_{0}(x), \quad t \geq 0, x \in \mathbb{R}^{n}
$$

satisfying for every $\varepsilon>0$

$$
|u(t, x)|=O\left(e^{\varepsilon|x|^{2}}\right), \quad|x| \rightarrow \infty .
$$

Next, assume that $\theta \in G^{\sigma}([0, \infty): \mathbb{R}), \sigma>0$, and for some $\nu \in(0,1], \theta \in S_{\nu}^{\sigma}\left(\overline{\mathbb{R}}_{+}\right)$ i.e. there exist two positive constants $A$ and $B$ such that

$$
\left|\theta^{(j)}(y)\right| \leq A^{j+1} j !^{\sigma} \exp \left\{-B y^{1 / \nu}\right\}
$$

for all $y \geq 0, j \in \mathbb{N}_{0}$. Then

$$
u_{0}(x)=\theta\left(x^{2}\right) \in S_{\nu / 2}^{\sigma+1-\nu / 2}\left(\mathbb{R}^{n}\right)
$$

and

$$
u(t, x) \in G^{2 \sigma+2-\nu}\left([0, \infty) ; S_{\nu / 2}^{\sigma+1-\nu / 2}\left(\mathbb{R}^{n}\right)\right) .
$$

In particular, if

$$
\theta(y)=\theta_{\varepsilon}(y)=\frac{1}{(4 \pi \varepsilon)^{n / 2}} e^{-y /(4 \varepsilon)}, \quad \varepsilon>0 .
$$

then

$$
u^{\varepsilon}(t, x)=\frac{1}{\left(4 \pi(t+\varepsilon)^{n / 2}\right.} e^{-x^{2} /(4(t+\varepsilon))}, \quad t \geq 0, x \in \mathbb{R}^{n} .
$$

solves (42) with initial data

$$
u^{\varepsilon}(0, x)=\frac{1}{(4 \pi \varepsilon)^{n / 2}} e^{-x^{2} /(4 \varepsilon)}, \quad x \in \mathbb{R}^{n} .
$$

Proof. The arguments are simple (as for the 2-D NS). By (45) and (46)

$$
\left|u_{0}(x)\right|=O\left(e^{\varepsilon|x|^{2}}\right), \quad \text { as }|x| \rightarrow \infty,
$$

for all $\varepsilon>0$, therefore $u(t, x)=e^{t \Delta} u_{0}$ is well defined and is a solution to the heat equation $\partial_{t} u-\Delta u=0$ with initial data $u_{0}$, unique in the class of functions satisfying (48). Next, we note that if $u_{0}(x)$ is radially symmetric, then $e^{t \Delta} u_{0}$ remains radially symmetric with respect to $x$ for all $t>0$ and by the hypothesis (43) we observe that $X_{j}\left(F_{j}\left(e^{t \Delta} u_{0}\right)\right) \equiv 0$.

We show the Gelfand-Shilov property for space dimension $n=1$, since the general case brings only notational complications. We have the following identity

$$
\begin{aligned}
\partial_{x}^{\alpha}\left(\theta\left(x^{2}\right)\right) & =\left.\sum_{j=1}^{\alpha} \frac{\theta^{(j)}\left(x^{2}\right)}{j !} \partial_{z}^{\alpha}\left(\left(z^{2}-x^{2}\right)^{j}\right)\right|_{z=x} \\
& =\left.\sum_{j=1}^{\alpha} \theta^{(j)}\left(x^{2}\right) \frac{\alpha !}{(\alpha-j) ! j !} \partial_{z}^{\alpha-j}\left((z+x)^{j}\right)\right|_{z=x} \\
& =\sum_{\alpha / 2 \leq j \leq \alpha} \theta^{(j)}\left(x^{2}\right) \frac{\alpha !}{(\alpha-j) ! j !} \frac{j !}{(2 j-\alpha) !}(2 x)^{2 j-\alpha} .
\end{aligned}
$$


Next, combining (56) with (49), we derive (see [4] for similar arguments for $\sigma=1 / 2$ ), by using the Stirling type formulas, the following estimates:

$$
\begin{aligned}
\left|\partial_{x}^{\alpha}\left(\theta\left(x^{2}\right)\right)\right| & \leq \sum_{\alpha / 2 \leq j \leq \alpha}\left|\theta^{(j)}\left(x^{2}\right)\right| \frac{\alpha !}{(\alpha-j) ! j !} 2^{2 j-\alpha} \frac{j !}{(2 j-\alpha) !}|x|^{2 j-\alpha} \\
& \leq \sum_{\alpha / 2 \leq j \leq \alpha} A^{j+1} j !^{\sigma} e^{-B|x|^{2 / \nu}} \frac{\alpha !}{(\alpha-j) ! j !} 2^{2 j-\alpha} \frac{j !}{(2 j-\alpha) !}|x|^{2 j-\alpha} \\
& \leq A \sum_{\alpha / 2 \leq j \leq \alpha}(4 A)^{j} j !^{\sigma} e^{-B|x|^{2 / \nu}} \frac{j !}{(2 j-\alpha) !}\left(|x|^{2 / \nu}\right)^{\nu j-\nu \alpha / 2} .
\end{aligned}
$$

Now we use $e^{-a z} z^{\gamma} \leq a^{-\gamma} \Gamma(\gamma+1)$ with $a=B / 2, z=|x|^{2 / \nu}, \gamma=\nu j-\nu \alpha / 2$ to get

$$
\begin{aligned}
& \left|\partial_{x}^{\alpha}\left(\theta\left(x^{2}\right)\right)\right| \\
& \quad \leq A \sum_{\alpha / 2 \leq j \leq \alpha}(2 / B)^{\nu j-\nu \alpha / 2}(4 A)^{j} j !^{\sigma} e^{-B / 2|x|^{2 / \nu}} \frac{j !}{(2 j-\alpha) !} \Gamma(\nu j-\nu \alpha / 2+1) \\
& \quad \leq A e^{-B / 2|x|^{2 / \nu}} \sum_{\alpha / 2 \leq j \leq \alpha}(2 / B)^{\nu j-\nu \alpha / 2}(8 A)^{j} j !^{\sigma}(\alpha-j) ! \Gamma(\nu j-\nu \alpha / 2+1) \\
& \quad \leq A C^{\alpha} e^{-B / 2|x|^{2 / \nu}} \sum_{\alpha / 2 \leq j \leq \alpha} j !^{\sigma}(\alpha-j) ! \Gamma(\nu j-\nu \alpha / 2+1) \\
& \quad \leq A C^{\alpha} e^{-B / 2|x|^{2 / \nu}} \sum_{\alpha / 2 \leq j \leq \alpha} j !^{\sigma} \Gamma((1-\nu / 2) \alpha-(1-\nu) j+1) ! \\
& \leq A C^{\alpha}(1+\alpha / 2) e^{-B / 2|x|^{2 / \nu}} \alpha !^{\sigma} \Gamma((1-\nu / 2) \alpha+1)
\end{aligned}
$$

for all $\alpha \in \mathbb{N}_{0}$, where $C=8 A(2 / B)^{\nu / 2}$. By the Stirling formula we get that (57) yields (50). As for (51), it follows from our linear estimates.

\section{References}

[1] H. Aikawa and N. Hayashi, Holomorphic solutions of semilinear heat equations, Complex Variables Theory Appl. 16 (1991), 115-125.

[2] H. A. Biagioni and T. Gramchev, Multidimensional Kuramoto- Sivashinsky type equations: singular initial data and analytic regularity, in: Proc. Fifth Workshop on Partial Differential Equations (Rio de Janeiro, 1997), Matemática Contemporânea 15 (1998), 21-42.

[3] H. A. Biagioni and T. Gramchev, Evolution PDE with elliptic dissipative terms: critical index for singular initial data, self-similar solutions and analytic regularity, C. R. Acad. Sci. Paris Sér. I 327 (1998), 41-46.

[4] H. A. Biagioni and T. Gramchev, Fractional derivative estimates in Gevrey spaces, global regularity and decay for solutions to semilinear equations in $\mathbb{R}^{n}$, J. Differential Equations 194 (2003), 140-165.

[5] G. Bourdaud, M. Reissig and W. Sickel, Hyperbolic equations, function spaces with exponential weights and Nemytskij operators, Ann. Mat. Pura Appl. (4) 182 (2003), 409-455. 
[6] A. Ferrari and E. Titi, Gevrey regularity for nonlinear analytic parabolic equations, Comm. Partial Differential Equations 23 (1998), 1-16.

[7] C. Foiaş and R. Temam, Gevrey class regularity for the solutions of the Navier-Stokes equations, J. Funct. Anal. 87 (1989), 359-369.

[8] I. M. Gel'fand and G. E. Shilov, Generalized Functions II, Academic Press, New York, 1968.

[9] M. Gevrey, Sur la nature analytique des solutions des équations aux dérivées partielles. Premier mémoire, Ann. Sci. École Norm. Sup. (3) 35 (1918), 129-190.

[10] T. Gramchev and G. Łysik, Sharp analytic-Gevrey regularity estimates down to $t=0$ for solutions to semilinear heat equations, Differential Integral Equations, to appear.

[11] T. Gramchev and L. Rodino, Solvability in Gevrey classes for semilinear PDE with multiple characteristics, Boll. Un. Mat. Ital. (8) 2-B (1999), 65-120.

[12] T. Gramchev and M. Yoshino, Rapidly convergent iteration method for simultaneous normal forms of commuting maps, Math. Zeitschrift 231 (1999), 745-770.

[13] Z. Grujić and I. Kukavica, Space analyticity for the Navier-Stokes and related equations with initial data in $L^{p}$, J. Funct. Anal. 152 (1998), 447-466.

[14] Z. Grujić and I. Kukavica, A remark on time-analyticity for the Kuramoto-Sivashinsky equation, Nonl. Anal. 52 (2003), 69-78.

[15] N. Hayashi, Analytic function spaces and their applications to nonlinear evolution equations, in: Analytic Extension Formulas and Their Applications (Fukuoka, 1999/Kyoto, 2000), Int. Soc. Anal. Appl. Comput. 9, Kluwer, Dordrecht, 2001, 73-86.

[16] K. Kobayashi, T. Siaro, and H. Tanaka, On the blowing up problem for semilinear heat equation, J. Math. Soc. Japan 29 (1977), 407-424.

[17] B. J. Levin, Distributions of Zeros of Entire Functions, Transl. Math. Monogr. 5, AMS, 1980.

[18] G. Łysik, Nonanalyticity of solutions to $\partial_{t} u=\partial_{x}^{2} u+u^{2}$, Colloq. Math. 95 (2003), 255-266.

[19] M. Mascarello and L. Rodino, Partial Differential Equations with Multiple Characteristics, Akademie Verlag - Wiley, Berlin, 1997.

[20] C. W. Oseen, Sur les formules de Green généralisées qui se présentent dans l'hydrodynamique et sur quelques-unes de leurs applications, Acta Math. 34 (1911), 205-284.

[21] S. Ouchi, On the analyticity in time of solutions of initial boundary value problems for semi-linear parabolic differential equations with monotone nonlinearity, J. Fac. Sci. Univ. Tokyo Ser. 1A 21 (1974), 19-41.

[22] S. Ouchi, Genuine solutions and formal solutions with Gevrey type estimates of nonlinear partial differential equations, J. Math. Sci. Univ. Tokyo 2 (1995), 375-417.

[23] L. Rodino, Linear Partial Differential Operators in Gevrey Spaces, World Scientific, River Edge, NJ, 1993.

[24] P. Takáč, P. Bollerman, A. Doelman, A. van Harten, and E. Titi, Analyticity for essentially bounded solutions to semilinear parabolic systems and validity of the Ginzburg-Landau equation, SIAM J. Math. Anal. 27 (1996), 424-448. 